$\xi=2$

\title{
The acoustic parameters for analysing speech with complete dentures
}

\author{
Gunasekar. $C^{1 *}$, Sabrigirinathan. $C^{2}$, Vinayagavel. $K^{3}$, Ramkumar. $\mathbf{K}^{4}$ \\ ${ }^{1}$ Research Scholar, HOD, Department. Of Dentistry, ESIC Medical college, Hospital \& \\ Post-Graduate Institute of Medical Science \& Research,TN, India \\ ${ }^{2}$ Guide, Professor, HOD. Department. of Prosthodontics Tamil Nadu government, Dental College \& Hospital, India \\ ${ }^{3}$ Professor, Department. of Prosthodontics, Tamil Nadu government Dental College \& Hospital, India \\ ${ }^{4}$ Research Scholar, Associate Professor. Department. of Prosthodontics, Stanley Medical College \& Hospital, India \\ *Corresponding author E-mail: cgsekar63@gmail.com
}

\begin{abstract}
The patients those who wear dentures have difficulty in speech becomes a major concern. Various studies were provided to improve speech with various technique, suggestions and conclusions but the better or exact test is to analyses the speech with complete dentures. Here we like to give brief details on understanding about proper knowledge on speech production, perception and the acoustic features and useful parameters to analyses these acoustic features with the quality of speech in denture wearers.
\end{abstract}

Keywords: Acoustic Analysis; Speech Articulation; Resonance; Formant; Palatal Augmentation

\section{Introduction}

Analysis of speech and sound production should be based on the knowledge about the nature of speech the sound themselves, the production of sound and the anatomic and physiologic structures involved. The purpose of this article is to provide the reasonable understanding of the procedures to analyses quality of speech in complete denture wearers. The objective to suggest some approaches focus on speech output acoustically, that is observed by the listener as well as the complete denture wearers, to analyses the acoustic features of formant frequencies, stop burst and voice onset time. This paper is a guide to the recent issues and literature on the acoustic analysis of speech with importance on Vocal Tract Acoustics.

\subsection{Background}

Scholosser and Gehl said that among the objectives for fabrication of denture prosthesis in amenability with phonetic requirements for correction of speech defects duly to complete loss of natural teeth was the $3^{\text {rd }}$ factor (Palmer 1974). Complete loss of teeth can cause a persistent speech disorder by altering dental articulation areas (Chierici \& Lawson 1973). It is necessary to cover the salient feature of the acoustic events (Palmer 1974) to improve the importance of the analytical process. In the event, the pressurized air, from lungs, passing through vocal folds gets vibrated and produces laryngeal tone. This voicing speech sound or the complete laryngeal tone passes through upper larynx, the pharynges. The mouth and sometimes nose got modified and resonated to produce speech sounds. Those speech sounds which have no laryngeal tone get modified with air flow and air pressure to produce standardized speech sounds (Chierici \& Lawson 1973). The air flow and air pressure above larynx are affected by structural elements like teeth, palate, lip, and tongue.
As the air obstructed through static and the dynamic structures create turbulence, these might be a narrow band of noise, called as speech sounds like fricatives (Palmer 1974) When this exhaled air meets complete abstraction, and the sudden release of noise produces the speech sound called as plosive or stops (Palmer 1974). The combination of friction and plosive with audible noise is called affricative. Examples of these voiceless speech sounds are: 1. Fricative- obstructed air flow: /s/, /sh/ 2. Plosive- Explosive air release: $/ \mathrm{p} /, / \mathrm{t} / / \mathrm{k} /$ and 3. Affricative- Explosive air release with friction: /ch/.

This same is used with addition of adjective 'voiced' 1. Fricativeobstructed air flow: /z/ 2. Plosive- Explosive air release: /b/ and 3. Affricative-Explosive air release with friction: /j/. Here both airs create noise and laryngeal tone (noise) (Palmer 1974).

Regardless of the importance of structural elements which are called static components to structural elements to articulate speech, there is a tremendously important dynamic component the tongue (Palmer 1974). The other dynamic components are velum, pharynx, lips and mandible. The exhaled airs from laryngeal noise are directed and controlled by ability by the dynamic structure tongue and is the basis in the formation of speech sounds. In the oral cavity to further understand the importance of adequate analysis, we should classify the process of the speech sound production. The tongue impeding the passage of air forced through a narrow space between the tongue apex and incisor teeth could be labelled interference and another mode, When the air is stopped, or dammed up and subsequently released could be termed interruption (Palmer 1974). The tongue is the integral part of the speech act, this dynamic structure in the traumatic events, aging, paresis tremor and other tongue aberrations. They make adequate adoption for the change in the tongue but when suddenly one finds with new structural environments like dentures, the learned compensator's movements no longer satisfy the requirements. The problem is not only the /s / sound, but sound such as the /l/, /t/ or / d/ also develop distortions. 


\section{Acoustic analysis}

The unique complex structure of the oral cavity is the main challenge to Prosthodontist. Apart from the aesthetic, functional aspects, we should focus on speech function and the morphologic or physiologic basis for assessment of its relationship to maintain acceptable speech.

Some patients (denture wearers) have difficulty in their speech, which becomes the major concern. The precise evaluation of speech problem is essential to provide quality dentures. One should delineate the difference of those speech defects which are associated with oral form and structural changes with other coexistent conditions in denture wearing patients.

Among the various dimensions basic to speech production to clarify areas of speech get affected due to complete denture is the concern for prosthodontist and general dental practitioners. The seven dimensions basic to speech are discussed by various author's (Chierici \& Lawson 1973). In that resonators and articulators are the main attention to analysis of the speech quality improvement in denture wearers.

\subsection{Resonance}

The air stream from the lungs passes through larynx which gets vibrated by vocal folds (cords) is not acoustic in nature. This laryngeal tone is augmented and modified by Pharynx, the oral cavity, and nasal cavity. These structures resonate from the air stream with alteration of their shape and size by neuromuscular action in coordination with soft palates, the tongue, cheek, jaws and lips to produce speech sounds (Palmer 1974).

The resonance balance between these structures is affected by dentures and is obviously a major concern in prosthodontic treatment. The congenital, acquired palatal defects and injury may also destruct the normal resonance and lead a decrease in voice quality (Palmer 1974).

For the complete denture wearers, there is a paucity of research findings on the acoustic effects of dentures. An experimental study by (Spangenberg 1937) reported that difference in resonance was associated with the alterations in the dimensions of the oral cavity which is produced by the palatal coverage of maxillary denture base. The thickness of dentures also affects voice quality, which reduces the size and shape in the oral cavity may affect the vocal tract resonance pattern (Tanaka 1973).

\subsection{Speech articulation}

Noise emerged from vibrated vocal cords and passes through the pharynx, oral and nasal route and radiate out from the mouth cannot be termed as speech (Chierici \& Lawson 1973). When, static and dynamic articulators contact either, by interference or interruption between them, that process in termed speech articulation (Chierici \& Lawson 1973). This articulation may also be affected by another condition like dysarthria's and neurologic impairment but edentulous patients, with normal anatomic and physiologic condition, are noticing the difficulties in articulation problems by their dentures.

The articulation defects from the oral structure like loss of teeth are directly concerned with removable prosthetics (denture) where the deformities comprehensively or relatively by teeth, lips, mandible, tongue hard and soft palates can affect accurate speech articulation (Chierici \& Lawson 1973).

Contact positions of the tongue relative to the palate and shape of the anterior palatal region are most conductive in the production of specific sound articulation i.e /s/ and /sh/ sounds. In addition to these fricative sounds like /s/ \& / sh/, other plosives /p/, /t/, /k/ and affricative $/ \mathrm{ch} /$ are distorted in complete denture wearers.

The anatomical feature of the palatal surface and the physical presence of maxillary denture may also retrieve sensory feedback and perception. The production of isolated speech sounds involves ten phonemes or sentence or word for the evolution of articulatory factors to produce consonant sounds. These are (Chierici \& Lawson 1973):

1) $/ \mathrm{p} /, / \mathrm{b} /, / \mathrm{m} /$ Bilabial contact

2) $/ \mathrm{k} /, / \mathrm{g} /, / \mathrm{ng} /$ velar contact

3) $/ \mathrm{t} / / \mathrm{d} /, / \mathrm{n} /$ palato-alveolar contact

4) $/ \mathrm{f} /, / \mathrm{v} /$ Labiodental contact

5) /th/ linguodental contact

6) $/ \mathrm{j} / / \mathrm{ch} /$ Palatal contact

7) /1/ Lateral lingual/palate lateral

8) /r/ Palato central contact

9) $/ \mathrm{s} /, / \mathrm{z} /$ Palato alveolar contact, and narrow air blade

10) $/ \mathrm{sh} /, / \mathrm{ch} /$ Palatal contact with wide air blade

Testing these sounds are the articulatory requirement for speech in complete denture construction. There are no specific test sounds for analysing speech universally, but it was recommended to have their own native language words or sentence or syllables into consideration. The importance of palatal rugae in relation to speech has not been established (Chierici \& Lawson 1973). Landa reported rugae in the denture is ineffectual and unnecessary thickness in the anterior palatal vault and detrimental to speech (Tanaka 1973). Recently, reproducing the rugae area in complete denture is recommended to improve the phonetic quality of complete denture (Zaki Mahross \& Baroudi 2015).

\subsection{Audition}

The physiological requirement for producing substantive acoustic signals, which are perceived and decoded by the listener is essential for speech communication. Distortions in speech articulation and quality are also associated with the reduction in hearing acuity (Chierici \& Lawson 1973). It is the evident that the denture wearer perceives his own speech differently from the listener's perception of it, on the process of hearing mechanism.

Neurological impairment can also lead to speech defects that may be cortical rather than dental. Apprehension prevents those denture wearers from the proper use of the speech mechanism.

\subsection{The acoustics underlying our observation about the speech}

To understand general principles of sound production in speech and to provide a single resource that give the good understanding of acoustics. Our purpose is to keep focus that ability analysis and interpret speech sound.

From the source of sound vibration, the vocal cord gets natural frequencies either transient or continuous, which is defined as resonance that will be amplified. Resonance in speech is called formants and they are labeled $\operatorname{asF}_{1}, \mathrm{~F}_{2}, \mathrm{~F}_{3}, \mathrm{~F}_{4}$ etc. starting with the lowest frequency.

Each resonance or formant is described in terms of its frequency amplitude and bandwidth. All the speech sounds are complex and have many frequencies. This complex wave is periodic and aperiodic that in speech has harmonic frequencies $\left(\mathrm{F}_{\mathrm{o}}\right)$ and fundamental frequencies (the waveform). Another type has non-integer multiple of lowest frequency voiced sounds, which are produced at the glottis vowels, nasal voice sounds and voice sounds produced above the glottis (friction noise) e.g. /f/,/s/.

Resonance is fundamental to speech acoustics because of difference in phonetic quality stalk in resonance patterns of vocal tract as it changes the shape (Fant 1971). Thus, we study the resonance properties of the vocal tract (VT) where length and shape are main determinant of the formants or resonance frequencies that can be seen and measured in the waveform, spectrogram which has a typical distinctive wave pattern.

\subsection{Formant}

Gunnar Fant (Fant 1971) defined the Formant: as 'The spectral peaks of the sound spectrum $P$ (f). This definition is the one broadly used in acoustics research and industry. For instance, a similar definition of formant by Benade (Podos et.al. 1995) state 
that: 'The peaks that are observed in the spectrum envelope are called formants. After defining formant, (Fant 1971). It then defines resonance frequencies of the vocal tract in terms of a gain function $T$ (f) of the vocal tract: The frequency location of a maximum' in T (f), i.e., the resonance frequency, is very close to the corresponding maximum in spectrum P (f) of the complete sound. There is even a third meaning in voice research. The acoustics of the vocal tract are often modelled Source Filter. The frequencies of the poles of this filter model fall close to those of the formants. As a result, some voice researchers now refer to the frequencies of the poles as formants. The formant refers to a peak in the spectrum (a property of the sound of the voice), to others it refers to a resonance of the vocal tract (a physical property of the tract), while to a third group it refers to the pole in a mathematical filter model (a property of a model).

In the broader field of acoustics, formant retains its original meaning: a broad peak in the spectral envelope of the sound (Epps et.al.1997). A formant is a concentration of acoustic energy around a particular frequency in the speech wave, and that is the dark band on a wide-band spectrogram, which corresponds to a vocal tract resonance. Technically, it represents a set of adjacent harmonics, which are boosted by a resonance in some part of the vocal tract. Thus, different vocal tract shapes will produce different formant patterns, regardless of what the source is doing. There are several formants, each at a different frequency, roughly one in each $1000 \mathrm{~Hz}$ band. There are a variety of studies showing various acoustic correlates of vowel quality, among them formant frequency, formant movement, and vowel duration. In that Formant, frequency (and movement) is probably the most important.

\subsection{Acoustic theory (source filter theory)}

Source energy from lungs at the glottis level gets acoustic pulses generated by vibrations of vocal folds that may be periodic, aperiodic spectrum or mixed (turbulence noise). Then these acoustic pulses travel through or transfer along the length and shape of vocal tract (VT) (Frequency-dependent transmission system) where it gets filtrated (resonated) at different area or shape of the VT, then reduced lower frequencies (radiation function) emerged out at the lips (Chierici \& Lawson 1973). The resonator acts as a filter on the original source of the sound.

The filter or transfer function of VT defined primarily is a resonance of VT, these resonances are called formants and each formant is specified by formant frequency (where $F_{o}$ is Formant number) and bandwidth. From vocal folds to mouth, there are infinite numbers of formants. But no more than 3-5 usually are considered in speech analysis. This formant is resonance properties of the VT. In the VT shape the complete pattern that can be estimated from measurement of the acoustic signals.

The resonance arising when, a wave of sound pressure (latent) or velocity (dynamic energy) traveling down along the vocal tract is called a traveling or plane wave. This can be compressed and expanded so that there are variations in sound pressure (latent energy). In speech, these different tonal qualities change depending on vocal tract configuration.

In speech, this source of sound is provided primarily by the vibration of the vocal folds. Vocal fold vibration is complex, consisting of both a fundamental frequency and harmonics. Because the harmonics always occur as integral multiples of the fundamental (x1, $\mathrm{x} 2, \mathrm{x} 3$, etc. The point is that vocal source isn't just one frequency, but many frequencies ranging from the fundamental all the way up to infinity, in principle, in integral multiples. Just as white light is many frequencies of light all mixed up together, so is the vocal source a spectrum of acoustic energy, going from low frequencies (the fundamental) to high frequencies. The energy provided by the source is then filtered or shaped by the body of the vocal tract (VT). In essence, the filter sifts the energy of some harmonics out (or at least down) while boosting others.

Understanding the speech articulation in oral cavity, which acts as resonator that modifies the laryngeal tone into speech sounds by blending with static articulator and dynamic articulators either by interference or interruption mode, when the complete denture placed in the mouth, especially the maxillary denture (Palmer 1974). The definite interplay between the structures and mode get affected and results in speech problems so main approach to the analysis of this problem is teeth, thickness of maxillary denture base, palatal contour or morphology.

\subsection{The problems}

In complete denture prosthesis, type of problems associated is generally articulatory. These errors are classified as omission, submission, and distortion. Probably the most common articulatory error in the complete denture is distortion. The speech sound error termed "Whistling S" or slushy speech (Palmer 1979). Distortions (lisping errors) occur in person with dysarthria, paralysis as well as complete denture wearers distorts them. The relationship of dentition to speech sound productions when the static and dynamic structures lack harmony to produce accepted noise exclusively when tooth position and covered palatal area are the structural deviation on speech sound distortion (Palmer 1979).

Regarding the concept of air turbulence, one might conclude that there is some role of palatal rugae in a speech as a point of contention. Recent studies conclude that rugae inclusion is essential for speech improvement in complete denture wearers (Zaki Mahross \& Baroudi 2015)

\section{Procedures in the acoustic analysis of speech}

Acoustic analysis may be classified broadly as time-domain, frequency-domain, and time-frequency domain analyses.

1) The waveform, or energy envelope of speech - time-domain analysis

2) Fast Fourier Transform (FFT) spectra, Linear Predictive Coding, (LPC) spectra, and the cepstrum- frequencydomain analysis

3) The spectrogram - time-frequency domain analysis

Acoustic data can be interpreted as deterministic or stochastic (Kent et.al. 1999). Individual spectral-temporal features, which relate to an aspect of speech production, are focused by Deterministic data. For example, formant frequency values pertaining to a particular vowel segment may be used to infer features of lingual articulation during that segment. Statistical features or indices typically collected over long time samples are by stochastic data, for example, calculation of a long-term average spectrum (LTAS) may be used to characterize the overall energy patterns for a particular speaking task.

The measurement of VOT (voice onset time) for voiced and voiceless stops as an index of the consonant voicing contrast in wordinitial position (phonetic aspects of speech) or an acoustic measure of laryngeal function (physiological subsystem components of speech production) can be interpreted with acoustic data (Kent et.al. 1999). (Fig: 01) Acoustic measurements can be quantified up to six acoustic features from the manually segmented sound recordings: 1. Formant frequencies and duration, 2. Voice onset time, 3. Hold phase, 4. Burst release, 5. Friction, 6. Pre-voicing durations. These quantitative measurements enabled statistical comparison between and within word-retrieval tasks, vowel frequencies (formants), and VCV syllables were measured at three points: F1, F2, F3 to allow comparison between consistency of vowel and consonants production. 

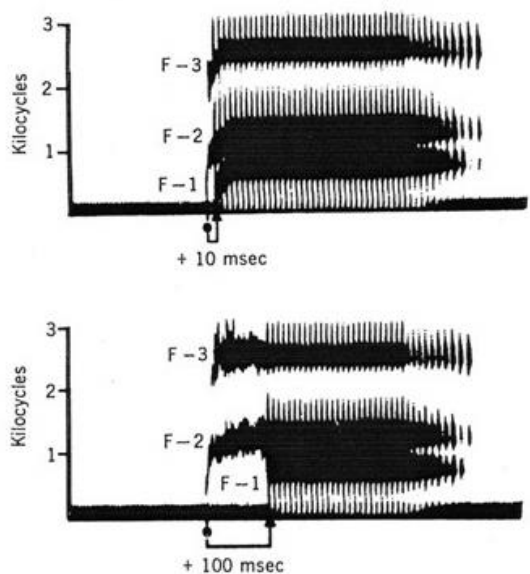

Fig. 1: Spectrogram of Synthetic Showing Two Values of Voicing Onset Time: A Small Voicing Lag Shown By [Ba] in Top Figure and Large Voicing Lag Shown By [Pa] and Formant F-1,F-2 and F-3 Represents Intense Bands of Energy in Speech Signal.

The frequency of any given formant has to do with the size and shape of the vocal tract. As the vocal tract changes shape, so do the formants change frequency. So the way the formants move into and out of consonant closures and vowel 'targets, is an important source of information about how the articulators are moving. It's worth pointing out now that all the formants show consonant transitions at the edges (Kent et.al. 1999).

\subsection{Instruments for phonetics analysis}

Among the instruments for the investigation of speech sound production are; the trained hearing of a speech and language professional, spectral analysis, and palatographics are the most important.

1) Palatography

2) Electromyography

3) Electrolaryngography

4) Electromagnetic Articulography (EMA)

5) Spectrography

\subsection{Spectrograms}

A graphic representation of three dimensions of sounds in terms of their component frequencies is called a spectrogram. In a spectrogram, time is always represented on the $\mathrm{x}$-axis and frequency on the y-axis. Intensity is depicted by the relative darkness of the frequencies shown. Formants can be seen in a wideband spectrogram, as dark bands.

A formant is a concentration of acoustic energy around a particular frequency in the speech wave. There are several formants, each at a different frequency, roughly one in each $1000 \mathrm{~Hz}$ band for average men. The corresponding range for average women is one formant every $1100 \mathrm{~Hz}$. The true range depends on the actual length of the vocal tract. Each formant corresponds to a resonance mode of the vocal tract.

Formants can be seen very clearly in a wideband spectrogram, where they are displayed as dark bands. The darker a formant is reproduced in the spectrogram, the stronger it is (the more energy there is around its frequency, or the more audible it is). When we look at a spectrogram, like this example (Fig: 02).

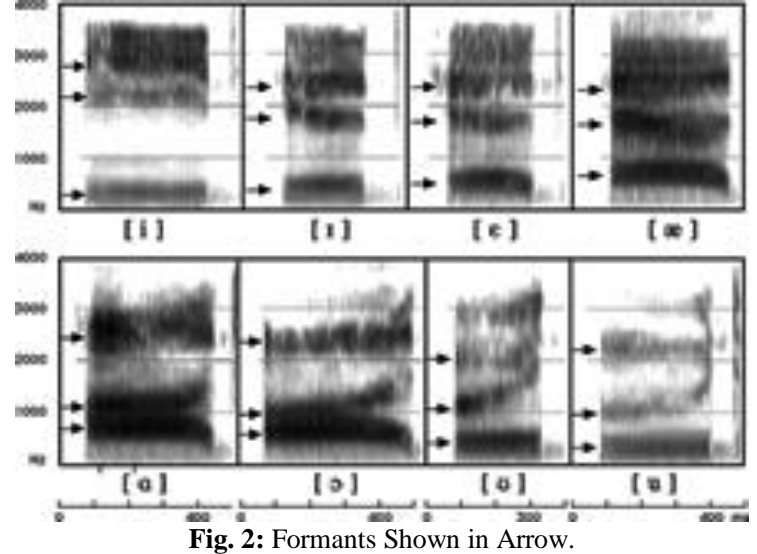

You will see formants everywhere, in both vowels and consonants. The vocal tract filters a source sound (e.g. periodic voice vibrations or aperiodic hissing) and the result of the filtering is the sound you can hear and record outside the lips and show on a spectrogram. To understand this, we must recall the source-filter theory of speech production. Formants occur, and are seen on spectrograms, around frequencies that correspond to the resonances of the vocal tract, i.e. at frequencies where the impedance is low (impedance is resistance to vibration at a given frequency) (Palmer 1974).

But there is a difference between oral vowels on the one hand, and consonants and nasal vowels on the other. For consonants, there are also anti-resonances in the vocal tract at one or more frequencies due to oral constrictions. Anti-resonance is the opposite of a resonance, such that the impedance is high rather than low at those frequencies.

Complex and non-periodical sound signals can be analysed using electronic computers and presented as a series of simple periodical signals of different frequencies and corresponding amplitudes, i.e. spectrograms (Kent 1993). Significant information about the quality of speech is provided by the shape of the spectrogram that contains quantitative data of a sound those strongly correlates to the speech system. Thus, they can be used for objective analysis of the speech system, for example, the phonetic quality of artificial dentures.

The following parameters are most useful for speech analysis in prosthodontics and orthodontic purpose for the acoustic features of sound measurement in which, suggested measures in Vowel Articulation the spectral correlates (Kent et.al. 1999): absolute values of $\mathrm{F}_{1}, \mathrm{~F}_{2}$, and $\mathrm{F}_{3}$ formant frequencies; $\mathrm{F}_{1}-\mathrm{F}_{\mathrm{o}}$ difference value; $\mathrm{F}_{2}$ $\mathrm{F}_{1}$ difference value; Spectral Correlates in Consonant Articulation and suggested measures are spectrum of stop burst or fricative noise, formant transitions for $\mathrm{CV}$ and VC transitions. Nearly all acoustic analyses can be classified (1) as to whether they are deterministic or stochastic, (2) by the number of variables used in the analysis, and (3) whether the analysis emphases on phonetic aspects, physiological components, both, or neither.

\subsection{Spectral envelope analysis}

A sound spectrogram (or sonogram) is a visual representation of an acoustic signal. The spectral envelope is analysed to yield the frequencies of a number of formants, specified by the user. A peak analysis is executed: depending on the parameters that were defined by the user, a number of resonators scan the spectral envelope.

\subsection{Analysis parameters}

Analysis Type

The spectral envelope can be calculated with two methods: LPC (linear predictive coding) and Discete Cepstrum, a Fast Fourier transform is applied to an electronically recorded sound. This analysis essentially separates the frequencies and amplitudes of its component simplex waves. The result can then be displayed visu- 
ally, with degrees of amplitude (represented light-to-dark, as in white=no energy, black=lots of energy), at various frequencies (usually on the vertical axis) by time (horizontal).

\subsection{Analysis order}

A filter has a central frequency, a bandwidth, a stop band with an attenuation rate, or slope. The analysis order represents the filter or resonator complexity. If our filter was a physical filter, this would represent the number of inductors and capacitors, which emphasize or filter the spectral components.

The filter's response is optimal with little energy for a given frequency, within a given passband. Once the limit of the passband is reached, its response decreases more or less progressively for neighbour partials: this characterizes the slope. The slope's steepness' depends on the filter's complexity. The more complex the filter, the steepest is the slope of the passband: the higher the attenuation rate is, as the frequency decreases.

\subsection{Relating vocal tract shape for acoustic output}

Sound production in an articulatory position, that guide to selected problems in a phonetic system especially by complete denture. Relating the source filter theory the consonants are classified according to the place of articulation, manner of articulation and voicing. To concise acoustic theory for consonants, the manner of articulation is taken as intern nasal consonants make that strong low - frequency acoustic energy introduces anti formants that reduce overall energy by absorbing damping over fleshy, convoluting lining mucosa of a nasal passage permit in the nasal radiation of sound energy (Kent 1993).

Fricative consonants depend on the size of constriction at the VT; the pressurized air driving through that constriction produces turbulence (Kent 1993). The two-way turbulence for fricative noise (Fig.03) generated by interruption and interference generally anterior constriction, shows spectral shaping of the fricative that is determined by the resonance stop consonants.
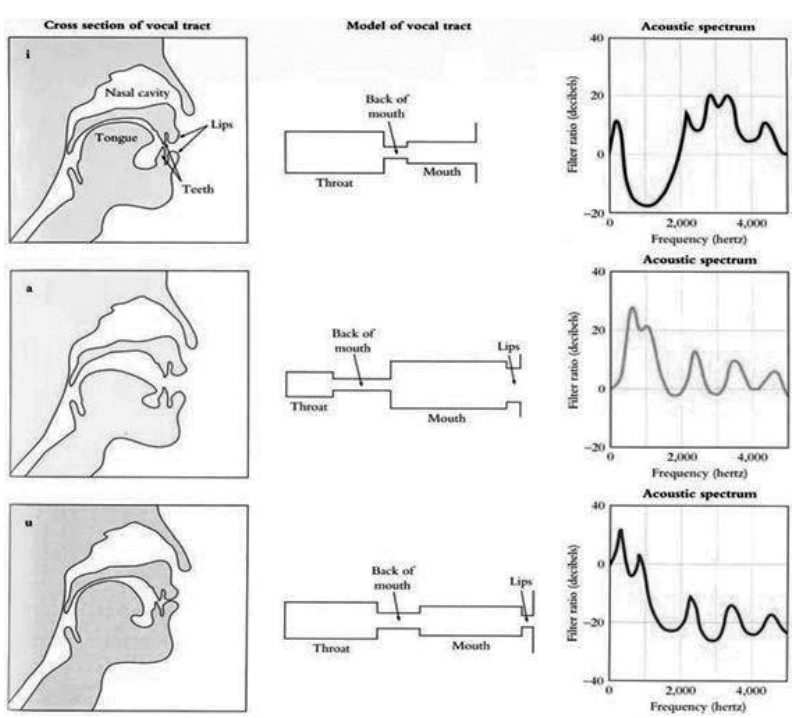

Fig. 3: The Two-Way Turbulence. Mode of Vocal Tract Showing the Pressurized Air Driving Through the Constriction at Back of Mouth and Lips.

The acoustic cues are some combination of a stop gap, release burst, and formant transition. The stop bursts are highly informative regarding the place of articulation and also important aspect of time-varying spectral features of burst. Cues to a place of articulation in stop consonants are Spectral energy distribution in the noise burst and formant transitions are the main cues. Formant transitions are context-sensitive cues.

Affricative consonants are a combination of stop and fricative articulations; liquid consonants are lateral and resembles nasal in having anti formants in the transfer function. This consonants /l/ and $/ \mathrm{r} /$ has formants structures by low, mid, high frequencies relatively to $\mathrm{F}_{1}, \mathrm{~F}_{2}, \mathrm{~F}_{3}$ ( about $600 \mathrm{~Hz}$ ) in that $/ \mathrm{r} /$ is distinguished from $/ \mathrm{l} /$, which have $\mathrm{F}_{3}-\mathrm{F}_{2}$ large difference (about $1500 \mathrm{~Hz}$ ). Vowel-like consonants are mostly the approximants; composed almost entirely of the formant transitions that also serve to identify their respective places of articulation.

Glide consonants are $/ \mathrm{j} /$ and $/ \mathrm{u} /$. The formant patterns for $/ \mathrm{j} /$ have similar to high front vowel /// and for /w/ similar to /u/ have a high back vowel.

Statistically analysing the acoustic features of speech sounds in complete denture wearer will not give significant results, when they are combining the total mean value and average value of both genders. The basic nature of the vocal tract configuration and the fundamental frequencies differ from male to female as well as for the child (Kent 1993). First of all, voice onset time (VOT), one of the most popular acquisition cues, was considered as a temporal gap between release of the burst in a stop consonant and onset of vowel (in msec) (Blumstein et.al.1977) and (Gandour \& Dardarananda 1984). VOT was visually defined as time between release of consonant burst and the first pulse (Stevens \& Klatt 1974) (starting point of vocal cycle within vowel) that also corresponded with beginning of formant transition time. Voice onset time and consonant duration were influenced by palatal augmentation (Arunoday Kumar et al. 2016).

\section{Conclusion}

Analysing the phonetic quality of artificial dentures from Speech spectrograms, would give as substantial information about the quality of speech and with computer analysis, it can be useful for the perceptual and objective analysis of the status within a speech system. However, the auditory and articulatory information is backed up by the spectrographic analysis is most important (Palmer 1979). The correlation between the quality of speech and palatal configuration is of importance in phonetic research in prosthodontics.

\section{Acknowledgement}

We readily acknowledge our indebtedness to Dr. G. Vijayabala, MDS, Dept. of Dentistry, ESIC Medical college, Hospital \& Post Graduate Institute of Medical Science \& Research.

\section{References}

[1] Palmer, J. M. (1974). Analysis of speech in prosthodontic practice. The Journal of prosthetic dentistry,31(6), 605-614 https://doi.org/10.1016/0022-3913(74)90115-2.

[2] Chierici, G., \& Lawson, L. (1973). Clinical speech considerations in prosthodontics: perspectives of the prosthodontist and speech pathologist. The Journal of prosthetic dentistry, 29(1), 29-39. https://doi.org/10.1016/0022-3913(73)90136-4.

[3] Palmer, J. M. (1979). Structural changes for speech improvement in complete upper denture fabrication. The Journal of prosthetic dentistry, 41(5), 507-510. https://doi.org/10.1016/0022 3913(79)90081-7.

[4] Spangenberg Jr, H. J. (1937). The effects of a dental prosthetic appliance on the quality of the voice: an experimental study. In Proc Staff Meet Mayo Clinic (Vol. 12, pp. 202-204).

[5] Tanaka, H. (1973). Speech patterns of edentulous patients and morphology of the palate in relation to phonetics. The Journal of prosthetic dentistry, 29(1), 16-28. https://doi.org/10.1016/00223913(73)90135-2.

[6] Fant, G. (1971). Acoustic theory of speech production: with calculations based on X-ray studies of Russian articulations (Vol. 2). Walter de Gruyter. https://doi.org/10.1515/9783110873429.

[7] Podos, J., Sherer, J. K., Peters, S., \& Nowicki, S. (1995). Ontogeny of vocal tract movements during song production in song sparrows. Animal Behaviour, $50(5)$ https://doi.org/10.1016/0003-3472(95)80044-1.

[8] Zaki Mahross, H., \& Baroudi, K. (2015). Spectrogram analysis of complete dentures with different thickness and palatal rugae mate- 
rials on speech production. International journal of dentistry, 2015. https://doi.org/10.1155/2015/606834.

[9] Epps, J., Smith, J. R., \& Wolfe, J. (1997). A novel instrument to measure acoustic resonances of the vocal tract during phonation. Measurement Science and Technology,8(10), 1112 https://doi.org/10.1088/0957-0233/8/10/012.

[10] Kent, R. D., Weismer, G., Kent, J. F., Vorperian, H. K., \& Duffy, J. R. (1999). Acoustic studies of dysarthric speech: Methods, progress, and potential. Journal of communication disorders, 32(3), 141-186. https://doi.org/10.1016/S0021-9924(99)00004-0.

[11] Kent, R. D. (1993). Vocal tract acoustics. Journal of Voice, 7(2), 97-117. https://doi.org/10.1016/S0892-1997(05)80339-X.

[12] Blumstein, S. E., Cooper, W. E., Zurif, E. B., \& Caramazza, A. (1977). The perception and production of voice-onset time in aphasia. Neuropsychologia, 15(3),

371-383. https://doi.org/10.1016/0028-3932(77)90089-6.

[13] Gandour, J., \& Dardarananda, R. (1984). Voice onset time in aphasia: Thai II. Production. Brain and Language, 23(2), 177-205. https://doi.org/10.1016/0093-934X(84)90063-4.

[14] Stevens, K. N., \& Klatt, D. H. (1974). Role of formant transitions in the voiced-voiceless distinction for stops. The Journal of the Acoustical Society of America, 55(3), 653-659. https://doi.org/10.1121/1.1914578.

[15] Arunoday Kumar et al. (2016) "Perceptual and acoustic analysis of speech with respect to posterior palatal seal area in complete denture prosthesis wearers", International Journal of Current Research, 8(5), 30759-30765. 